\title{
Not so Great Attractor?
}

Recent cosmological surveys suggest a wholesale streaming motion of galaxies across the sky, conflicting with the idea that local galaxies move under the influence of a Great Attractor. But the issue is not settled.

Four years have passed since Donald Lynden-Bell and six colleagues described the concerted motion of about 400 elliptical galaxies towards the Centaurus region, in excess of their expected cosmological recession (Astrophys. J. 326, 19; 1988). Later, Alan Dressler and Sandra Faber concluded that more distant galaxies in the same direction were receding less rapidly than cosmological expansion would require (Astrophys. J. Lett. 354, 45; 1990). The conclusion seemed obvious, if surprising: a huge concentration of mass exists, sufficient locally to distort the Hubble expansion and to give galaxies peculiar motions of hundreds of kilometres a second. That was the Great Attractor.

Now, D. S. Mathewson, V. L. Ford and M. Buchhorn (Astrophys. J. Lett. 389, 5; 1992) have published their own survey of galactic velocities and distances in the same patch of sky, but have come to a quite different conclusion: there is no Great Attractor. They say that the galaxies beyond the supposed Great Attractor are not falling back, but are moving away as rapidly as the foreground galaxies.

Adding to the confusion is a survey by J. Willick (Astrophys. J. Lett. 351, 5; 1990) of galaxies in the Perseus-Pisces cluster, almost antipodean to the Great Attractor. Willick found that galaxies there are moving towards us, and so in the same direction as the wholesale motion found by Mathewson et al. In other words, all the galaxies from the Perseus-Pisces region on one side of us to the Centaurus region on the other are streaming across the Universe with a collective velocity of more than $500 \mathrm{~km} \mathrm{~s}^{-1}$. If the Great Attractor does not exist, can there be an Even Greater Attractor further away still?

There is an even more bizarre interpretation. The cosmic microwave background has a substantial dipole moment, which is taken to mean that we are moving at about $600 \mathrm{~km} \mathrm{~s}^{-1}$ relative to a cosmic standard of rest in which the microwave background would be perfectly isotropic. It happens that this velocity is roughly consistent with the velocity at which Lynden-Ball et al. said we are moving towards the Great Attractor. This is tidy, meaning that the Great Attractor is more or less stationary in the cosmic rest frame, and that we and all the other galaxies in our neighbourhood are falling towards it.

But, if Mathewson et al. are right, that will no longer wash. Instead, they say that the apparent streaming motion of galaxies (our own included) across the entire sky arises only because all the velocities have been referred to the supposed cosmic rest frame. So the data can be given quite a different meaning: if our galaxy is taken to be 'at rest', so are all the others, and the streaming disappears. But if we are not moving with respect to the microwave background, the microwave background must be moving with respect to us. It is possible to make cosmological models with an apparent anisotropic cosmic motion of this sort, but the average cosmologist would find them unappealing.

Fortunately (or unfortunately, for those who prefer the bizarre to the sensible), the original answer may be correct. Faber has pored over the Mathewson survey; she does not dispute the data, but is less than convinced by the interpretation. All such surveys must contend with the age-old problem of observational cosmology: redshifts (velocities) are easy to measure, distances are difficult. The redshift of any galaxy is composed of cosmological recession plus peculiar motion, and if the distance is independently known, the cosmological part of the redshift can be subtracted away, leaving only the peculiar motion.

There is, it happens, a fairly tight correlation between the absolute luminosity of galaxies and the velocity dispersion of stars within them, which can be measured by the width of a suitable emission line. Therefore, measure the velocity dispersion, estimate the absolute galactic luminosity from the known correlation, compare it with measured luminosity to get distance, figure out the cosmological redshift for that distance, subtract from the measured redshift, and find the peculiar velocity. By this procedure, peculiar velocities can be mapped out and attractors great and small, or large-scale streaming motions, show up.

If all galaxies were identical this would be easy. But the correlation between luminosity and velocity dispersion has some scatter, which translates into errors in distance determinations. This would still not be too bad, were it not for another pernicious effect: bias. Brighter galaxies are the more prominent, and so are the more likely to be included among the high redshift objects in deep galaxy surveys. Such a bias towards the higher intrinsic magnitudes is more than a nuisance, because distance determination itself depends on luminosity. So there is potential for all kinds of subtle systematic biases, and any bias that can in principle occur will do so. So much has been known almost since observational cosmology began, but coping with bias still engenders argument.

Faber, speaking at a recent National Academy of Sciences colloquium at Irvine, California, said she believes that a proper accounting for bias would make the survey of Mathewson et al. consistent with her earlier work with Dressler, in which the far-side infall to the Great Attractor was established: Mathewson et al. provide a survey of galaxies in an 'ordinary' direction as well as towards the Great Attractor, and subtracting one from the other, to erase directly any systematic redshift-dependent effects, shows that the pattern of motion is indeed towards a relatively nearby centre, not an overall streaming.

In most galaxy surveys, independent distance estimates are unavailable, and only the redshifts are measured. In the absence of further information it would be impossible to separate the redshift into its cosmological and peculiar parts, but surveys also record the positions of galaxies. If peculiar velocities are due solely to the gravitational influence of galaxy clusters, then galaxy positions and velocities are related (by Poisson's equation for the newtonian gravitational potential), and with a sufficiently complete survey it becomes possible to analyse simultaneously the positions and redshifts of galaxies so as to reach a self-consistent picture yielding the peculiar velocities.

Michael Rowan-Robinson, discussing at Irvine such an analysis of the IRAS (Infra-Red Astronomy Satellite) sky survey, professed himself satisfied that all is more or less well. The most prominent large-scale peculiar galactic motions are reasonably well accounted for by the largest galaxy clusters. But one discrepancy stands out: the region between us and Perseus-Pisces is relatively empty, which would suggest that galaxies in the PerseusPisces region ought to be moving away from us. In fact, as Willick found, they are moving towards us. Rowan-Robinson thinks this will be resolved with more comprehensive surveys, but, in the light of Mathewson et al., there is a lingering suspicion that something funny may be happening along the Perseus-Pisces/Centaurus axis. A few more years should tell us whether the Universe is really uniform and isotropic, in the large, or if relativists ought to start dusting off their anisotropic cosmologies.

David Lindley 